\title{
PROTECTIVE EFFECTS OF SULFORAPHANE ON THE ALCOHOL-INDUCED TOXICITY AND ENDOPLASMIC RETICULUM STRESS IN C57BL/6 MICE
}

\author{
Baolong Li1, Yaojie Zhao1, Lina Luo1, Shaodong Wang1, Peng Lei2, Yujuan Shan2
}

1Center of drug safety evaluation, Heilongjinag University of Chinese medicine (No. 25 Heping Road, Xiangfang District, Harbin, China, 150040)

2 Department of Food Science, Harbin Institute of Technology (No. 73 Huanghe Road, Nangang District, Habin, China, 150090) E-mail: Ibl73@163.com; shanyujuan@hit.edu.cn

\begin{abstract}
Objective It is known that alcoholic liver disease (ALD) caused by the acute and chronic exposure to alcohol is one of the chronic liver diseases with the high mortality. Sulforaphane (SFN), rich in various cruciferous vegetables, has been widely studied as a chemo-preventive agent. In this study, we focused on the protective effects of SFN on alcohol-induced toxicity and mechanisms. Methods Male C57BL/ 6 mice were orally administrated with SFN $(0,12,40,80$ $\mathrm{mg} / \mathrm{kg} . \mathrm{bw}$ ) for 14 days. At the 13 th day, mice were challenged with alcohol ( $5 \mathrm{~g} / \mathrm{kg} . \mathrm{bw})$ every $12 \mathrm{~h}$ for 3 times. Results SFN markedly reversed the alcohol induced decrease of antioxidant capacity through enhancing GSH, GSH-Px, GST redox system. And the protective actions are related with activating Nrf2 and inactivating NF-KB. SFN attenuated the triglyceride (TG) and cholesterol (CHOL) contents which is possibly mediated by down-regulating sterol regulatory element-binding protein-1 (SREBP-1C). In addition, SFN weakened the stimulation of the chaperone GRP78, and its downstream sensory receptor ATF-6, triggered by acute alcohol intake. Conclusion The results suggest that antioxygenation is involved in the protective effects of Sulforaphane on the alcohol-induced toxicity. The current findings indicated that SFN weakened the liver toxicity triggered by alcohol through enhancing antioxidant capacity and reducing the endoplasmic reticulum stress.
\end{abstract}

Keywords: sulforaphane; alcoholic liver disease; endoplasmic reticulum stress

UDC 577.2616-082:616-035.1 DOI 10.22448/AMJ.2017.3.13-15

\section{PERSONIFIED MEDICINE - MEDICINE OF THE 21ST CENTURY}

\section{E.A. Borodin}

\section{Amur State Medical Academy, Russia}

In April 1953, the journal «Nature» published an article by James Watson and Francis Crick, "The Molecular Structure of Nucleic Acids, the Structure of Deoxyribonucleic Acid" (1). It is with this publication that the emergence of a new biological science - molecular biology - the science of the molecular foundations of life, is connected. mechanisms of storage, reproduction, transfer and realization of genetic information, structure and functions of molecules of biopolymers - nucleic acids and proteins (2). The paper proposed a three-dimensional spatial model of a DNA molecule in the form of a double helix. From the model follows the principle of matrix synthesis, i.e. syntheses, in which information about the structure of the synthesized molecule is encoded in the structure of the molecule-matrix. The mRNA molecule is synthesized on the matrix of the DNA molecule, which in turn is the matrix for the protein-synthesized polypeptide chain synthesized on the ribosomes. Thanks to this discovery and the suggested direction of reading the genetic information of RNA DNA, the protein, called the central dogma of molecular biology, was universally recognized. Molecular biology uses its own methods of research - genetic engineering, cloning of cells and organisms, artificial expression and knockout of genes. In the 20th century, molecular biology achieved tremendous results, explaining the molecular foundations of the most important manifestations of vital activity-the storage and transfer of genetic information, immunity, cellular respiration, apoptosis, and others, and the molecular mechanisms of the origin of the most important human diseases.

The term "molecular illness" was first used in 1949. Linus Polling applied to sickle cell anemia, a disease caused by a point mutation accompanied by the replacement of negatively charged glutamic acid in the 6th position of the hemoglobin $\beta$ chain with a hydrophobic amino acid valine and leading to a sharp decrease in the solubility of hemoglobin, precipitation, change in the shape of erythrocytes and restriction The ability of cells to carry oxygen (3).

The starting point for the emergence of new directions of medicine, for example gene therapy, therapeutic cloning, which allows to receive stem cells by genetic engineering methods and use them as medicines, was the implementation of the international scientific project "Human Genome" (1989-2002). The project was aimed at complete DNA sequencing of Homo sapiens ie. establishment of a sequence of 3.2109 pairs of nucleotides in a DNA molecule. The project was conceived in the mid-80s of the 20th century and began to be implemented since 1990. Initially, the scientists believed that the project would take a whole century to complete, then, taking into account the unprecedented methodological progress, the project was planned to be completed by 2005 , but in fact the main goals were achieved by mid-2001. And the organizers of the project reported on its more than successful implementation. The implementation of the project provided detailed information on the structure, organization and functioning of human DNA, contributed to the development of new effective technologies in the field of molecular biology, the creation of international electronic databases of genes and proteins open for free access, led to the emergence of a new triad of biological sciences - genomics, proteomics and bioinformatics, Had immense significance for biology, medicine and the international community as a whole and each individual, opened up new per- 